\title{
Saving and Restoring Mechanisms for Tangible User Interfaces through Tangible Active Objects
}

\author{
Eckard Riedenklau, Thomas Hermann, Helge Ritter \\ Ambient Intelligence / Neuroinformatics Group, CITEC, Bielefeld University, \\ Universitätsstraße 21-23, 33615 Bielefeld, Germany \\ [eriedenk, thermann, helge]@techfak.uni-bielefeld.de
}

\begin{abstract}
In this paper we present a proof of concept for saving and restoring mechanisms for Tangible User Interfaces (TUIs). We describe our actuated Tangible Active Objects (TAOs) and explain the design which allows equal user access to a dial-based fully tangible actuated menu metaphor. We present a new application extending an existing TUI for interactive sonification of process data with saving and restoring mechanisms and we outline another application proposal for family therapists.
\end{abstract}

Keywords: Human-Computer Interaction, Tangible User Interfaces, actuated Tangible Objects, Tangible Active Objects, Save and Restore, Menus

\section{Introduction}

Nowadays computers and the internet offer a rapidly increasing number of programs and functions with increasing complexity for almost every purpose. Human-Computer Interaction (HCI) becomes more and more important to find understandable interaction metaphors to these functions more user-friendly. The standard computer workplace consisting of display, keyboard and mouse is not satisfactory on some occasions. Tangible User Interfaces (TUIs) here offer new interaction metaphors by linking digital information to physical objects to make these digital becomes manipulable by multiple users with their everyday manipulation skills. Since many TUIs use motionless 'passive' objects, even basic standard functions we are familiar with from Graphical User Interfaces (GUIs), such as saving and restoring are difficult to implement. In this paper we present actuated Tangible User Interface Objects (TUIOs) that allow implementing these functionalities. 
After a short introduction to table-top TUIs and a problem statement we give an overview of the state of the art in actuated TUIOs. In Sections 3 and 4 we describe our developed hardware and software. We explain our Save \& Restore approach in detail in Section 4 and we present its application to an interactive sonification system. We end our paper with a short discussion and conclusion with suggestions for future work.

\subsection{Table-top Tangible User Interfaces}

Table-top TUI systems in general consist of a surface equipped with some sensing systems for object detection. This can be achieved by visual tracking through a glass surface but there are alternative techniques, such as radio frequency (RF) tracking as used in the AudioPad (1). TUIOs are often simple geometric shapes equipped with markers for tracking. Some systems also allow the visual projection of additional information onto the surface either from above or underneath. Also additional input techniques, such as touch input are also realized in some systems through special visual markers (2), or multi-touch via through Frustrated Total Internal Reflection (FTIR) surfaces, a method originally proposed by Han (3), to name a few. Beyond touch there are systems that also incorporate sound input and output such as the reacTable (2) or AudioPad.

Most table-top TUIs are incapable of saving and restoring arrangements or configurations of TUIOs on the table-top surface. The user either has to remember the arrangement himself, or may take a photo of it and for reconstruction he usually has to reposition the objects manually. To allow the system to save and restore the arrangement of the TUIOs, it needs to be enabled to control them actively. In the following section we will briefly describe existing systems with such actuation possibilities.

\section{State of the art in actuated TUIs}

There already exist systems that cope with the saving and restoring problem or are at least technically able to solve it. PSyBench, the Physically Synchronized Bench from the MIT Media Laboratory is the oldest one (4). Here the positions of objects can be synchronized between two 'coupled systems' by a magnetic 2-axis positioning mechanism combined with position sensing realized by a grid of membrane switches. Because of the mechanical design the objects can only be moved one after another.

Another system from MIT Media Laboratory is the Actuated Workbench (5). In this system a special surface is equipped with a grid of individually controllable electromagnets which allow moving ferromagnetic objects around on the surface very quickly. The objects contain a battery and LED for visual tracking from above. Beside classical functions of TUIOs, such as sorting, the authors consider basic functions, e.g. 'undo', or teaching and guiding. Furthermore they propose higher level applications, including remote collaboration, simulation and entertainment. 
PSyBench and the Actuated Workbench do not allow controlling the orientation of the objects. Weiss et. al. (6) extended the technology used in the Actuated Workbench by using objects equipped with multiple magnets. This allows rotating the objects and controlling properties of the widget like objects, "Madgets", such as buttons, dials, etc.

The above mentioned projects all use special yet rather low-cost objects. The main disadvantage of this technology is the dependence on a specially constructed and thus expensive surface.

The Planar Manipulator Display (PMD) by Rosenfeld et. al. (7) is another interesting approach for actuating objects. It is technically quite similar to our approach, since they use small-sized mobile robotic platforms. In contrast to our approach, the PMD small-sized mobile robots are slightly bigger than our TAOs and do not represent data themselves, but carry objects they move around. Rosenfeld et. al. also proposed a menu interface to save and restore different arrangements of the objects on the table. The menu used in the PMD system is projected on one side of the interactive surface.

All these systems technically allow saving and restoring arrangements of their actuated TUIOs. Only the PMD offers an implementation for this approach whereas Pangaro et. al. only consider saving and restoring mechanisms for future developments of the Actuated Workbench without offering concrete implementation details.

\section{Tangible Active Objects}

In this paper we present our Tangible Active Objects (TAOs) (8) and propose a way of tangibly managing multiple object arrangements. Like the objects in the PMD, our TAOs are small-sized mobile robotic TUIOs, which are able to move around on the interactive surface of our tDesk (formerly known as Gesture Desk (9)). The tDesk is equipped with a glass surface and a Firewire camera placed underneath the surface for visual tracking of objects. It is our base platform for table-top TUIs, as shown in Fig. 1.

\subsection{Hardware}

To build the TAOs' housings we utilized TUImod (10), modular combinable building blocks for TUIOs which were fabricated using a rapid prototyping printer. The electronics are organized in layers with different functionalities. They are physically interconnected through vertical buses which make the design modular and open for future extensions. The main layer is built around the Arduino mini pro ${ }^{1}$ platform, a rapid prototyping platform for electronics using an ATmega138 microcontroller. To establish a wireless connection between the TAOs and the host computer another

${ }^{1}$ http://www.arduino.cc/en/Main/ArduinoBoardProMini 
layer was designed carrying an XBee module ${ }^{2}$. The differential drive is controlled by an $\mathrm{H}$-bridge. Between the wheels of the drive a marker is embedded for visual tracking. Fig. 1 depicts the tDesk and the TAOs.
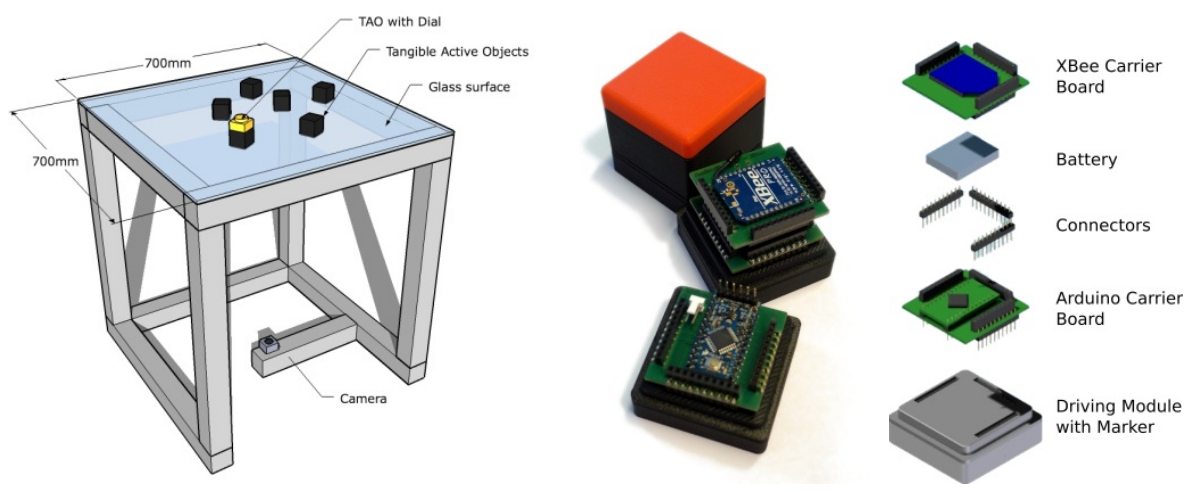

Fig. 1. a) The left most picture depicts the tDesk hardware setup consisting of a cubical table with an edge length of $70 \mathrm{~cm}$ which is equipped with a glass surface and a Firewire camera for tracking the TAOs from underneath the surface. b) In the center the manufactured devices are shown at different assembly stages (without batteries). c) The right picture shows an explosion drawing of the standard hardware architecture.

\subsection{Software Architecture}

The TAOs are controlled by a distributed system of software modules, communicating over the XML enabled Communication Framework (XCF) (11), a powerful high-level communication middleware. Fig. 2 depicts an overview over the modules and their communication relations.

The base system is organized in a closed control loop (gray arrows in Fig. 2) starting with the vision module which processes the captured camera images and extracts the marker information (2D position, orientation, and ID) of the TAOs. This marker information is spread over the middleware, connecting the software modules. Other software modules can subscribe to certain information patterns and are notified whenever matching information come in. This content-based notification mechanism allows a loose coupling between the software components. Along with the application modules which are described in the following section, the path planning module subscribes to the visual marker information and computes trajectories and navigation commands which a relay module transmits wirelessly to the TAOs. The trajectories are computed using gradient descent in a potential function whose minimum is located at the target location. Obstacles, such as other TAOs, are automatically avoided by adding repulsive potential hills centered dynamically at obstacle locations. Each step in turn results in a changed camera image and changed marker information and the control loop is closed.

${ }^{2}$ http://www.digi.com/products/wireless/point-multipoint/xbee-series1-module.jsp 


\section{$4 \quad$ Persistent active user interfaces}

Our first attempt to solve the problem of saving and restoring TAO configurations incorporated an RFID reader on one side of the tDesk and TAOs equipped with RFID tags. The main problem with this approach is that the reader is stationary and not equally accessible by every user. So we changed our implementation and present a new strategy to equip existing TUIs incapable of saving and restoring their configuration. We use two different hardware configurations of TAOs in this approach. The first type of TAOs, depicted in Fig. 1, is the standard configuration consisting of drive, main, and wireless layer. These TAOs are used to interact with the underlying TUI system itself and are referred to as Interaction TAOs (iTAOs) in the following.

The dial layer is used in the second type of TAOs depicted in Fig. 3. These objects identified via a Fiducial marker, and do not have the differential drive of the iTAOs. So these TAOs cannot move around on the table-top surface and their marker information is not passed to the underlying TUI since their position is not relevant neither for the menu controlling nor the TUI. This TAO class can be easily slid over the surface by the user and even other moving TAOs. In the following we refer to these TAOs as Menu TAOs (mTAOs).

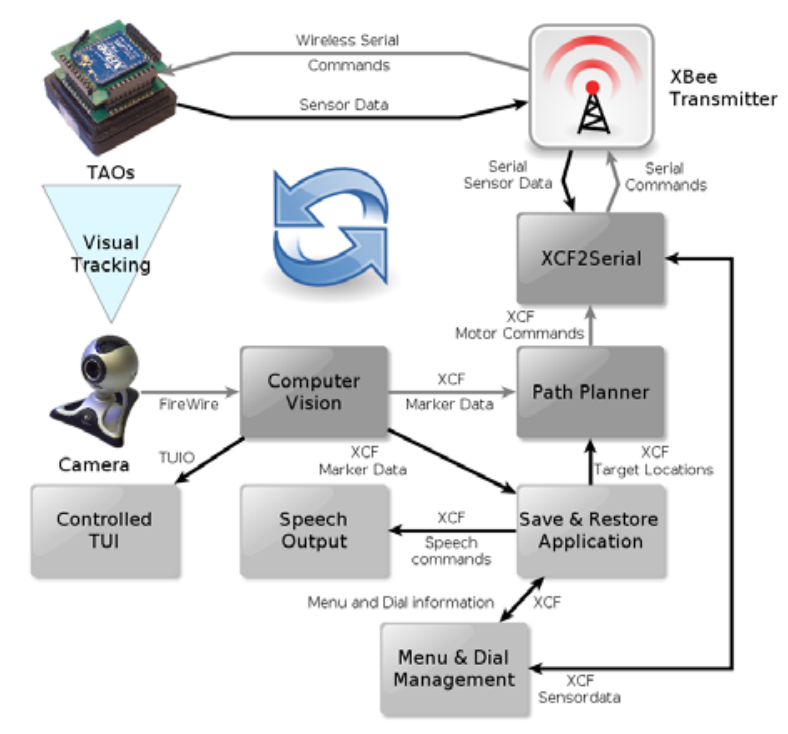

Fig. 2. The software modules and their communication relations build a closed control loop.

To create a fully tangible actuated menu metaphor for persistent tangible representations, we created a new hardware layer with an actuated dial set-top for the TAO which is able to represent different states with an internal degree of freedom and thereby allows the user to select different commands as shown in Fig. 3. The dial is implemented using a motorized potentiometer with $300^{\circ}$ rotational angle. Therefore it 
can act simultaneously both as input and an output device and adds an internal degree of freedom to our TAOs. We added basic speech synthesis to convey the menu structure to the user. Moving the menu dial from left to right, a spoken voice feedback reports each menu item. An item is selected by letting the dial rest on the actual item for a few seconds. Again feedback informs the user about the action selection and eventually the dial state changes.

For a tangible representation of TAO arrangements we propose to apply the concept of physical containers, introduced in the mediaBlocks project (12). Menu TAOs with a dial can be associated with the arrangement of the first type of TAOs on the table by selecting the "save" command from the dial menu. Of course it is also possible to restore an arrangement associated with a menu TAO by selecting the "restore" command with the dial. The menu is context sensitive depending on the association state of the actual mTAO. An unassigned mTAO has only two menu items: "Empty" is in the left half of the rotation range, and "save" is in the right half. If the mTAO contains a stored configuration it possesses a different menu: "Charged", "reconstruct", and "delete" cover each one third of the dial's rotation range. Fig. 4 depicts the two context-dependent menu structures. The motorized potentiometer allows to represent the association state of the mTAO. Depending on whether the mTAO has an associated arrangement or not, the menu has the items of the unassigned or assigned state and is physically set to the "empty" or "charged" item when the mTAO is put on the table-top surface.
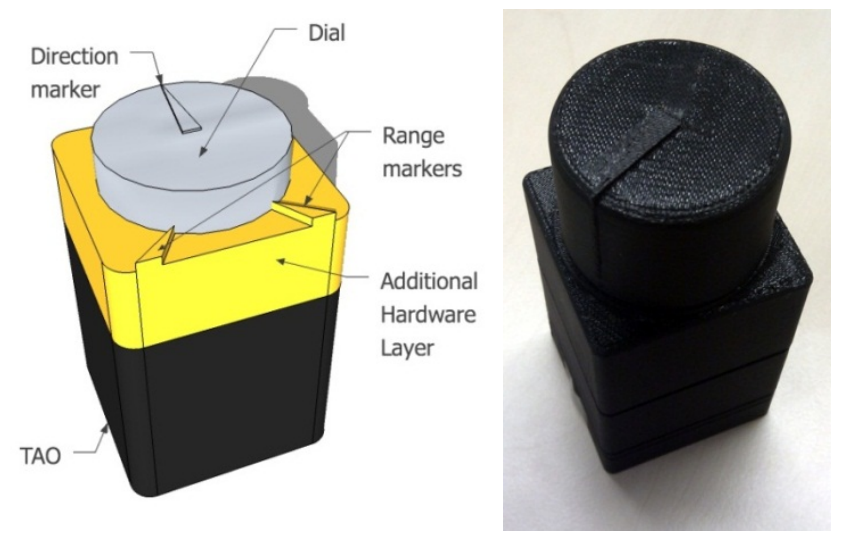

Fig. 3. Assembled second TAO type with menu dial (mTAO). The right picture shows a first working prototype of this new hardware component.

This setup allows extending existing TUIs with the ability to manage the arrangements of all involved TUIOs, as long as the functionality of these TUIOs is static and not changeable during interaction. 


\subsection{Integration of the active TAO menus into passive TUIs}

In order to integrate our approach into any existing TUI incapable of saving and restoring arrangements, the marker information of the TAOs needs to be made available to the control system. For this the TAO vision module offers output of the marker information using the TUIO protocol described in (13) which is already used by many TUIs. In this case there is no need for an additional camera and the positions are exactly the same in the extended TUI and the TAO system.

As depicted in Fig. 2, the save-and-restore module can be invoked to save the current positions of the TAOs and also to restore them by navigating the TAOs to new target locations. To control these actions, the users simply put an mTAO on the tabletop surface. If the TAO does not carry an assigned configuration, the dial automatically rotates left to represent the "empty" state according to the menu layout depicted in Fig. 4. To save a configuration, the user rotates the dial rightwards to the "save" menu item. Thereby the mTAO physically represents that it now carries a configuration and the new menu layout as shown on the right in Fig. 4 is active with respect to the new state. Verbal feedback indicates successful state changes. Correspondingly the dial rotates right to the "charged" state if a mTAO carrying a configuration is put on the table. To reconstruct the arrangement the user rotates the menu dial to the "reconstruct" menu item. The iTAOs then start to navigate to the new target locations and the TAO dial rotates back to the "charged" state. The users also have the possibility to delete the content of a menu TAO by rotating the menu dial left to the "delete" menu item. Thereby the "empty" state is directly represented. State changes and triggering commands by selecting the particular menu item also results in vocal feedback.
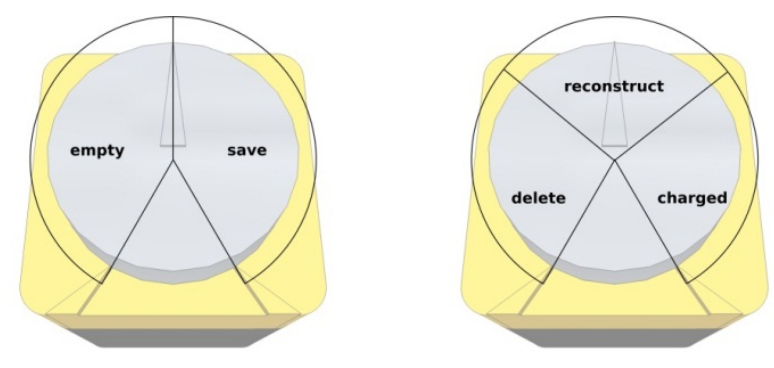

Fig. 4. Menu layouts for the "empty" and the "charged" state.

\section{$5 \quad$ Application examples}

As proof of concept we demonstrate our system in conjunction with an existing TUI for an interactive sonification of traffic data. Furthermore we propose a second application for family therapist which we consider for follow-up implementation. 


\subsection{Working with a tangible interface for an interactive sonification}

As first application, we demonstrate our approach to save and restore TUIs at hand of SoundBlox ${ }^{3}$ project, a TUI for the interactive sonification of traffic data, or more precisely, a vehicle registration station at a highway where for each car, bus or lorry certain features such as time, velocity etc. are recorded. Sonification is the auditory representation of data, allowing for instance monitoring of processes without the need to attend any visual display. In SoundBlox, TUIOs represent acoustic parameters such as pitch, level or brightness of short sound events. On the desk surface, specific fixed locations are defined to represent features of the data set such as the vehicle velocity, vehicle type, distance to the vehicle before, etc. By arranging the TUIOs on the desk, the user can now interactively control how data features are represented by sound features in the real-time rendered data sonification. For instance, moving the pitch object closer to the velocity location strengthens the connection so that faster vehicles become audible as higher pitched sounds. In search of suitable sonifications, where for instance traffic jams and fluent traffic can be better discerned, users wish to record the object arrangement as a preset to be used later, e.g. to compare the sound of different configurations. To this end, our mTAO can easily be applied. Since the mTAO can be passed between users, this allows several users to work together. The system even allows multiple mTAOs to store several presets at the same time. A video demonstration of this application is provided on our website. ${ }^{4}$

\subsection{Therapist assistance for measuring family relationships}

As promising application area we propose to use our approach for the problem of measuring family relationships as described in the Family-System-Test (FAST) by Gehring et. al. (14). In this method patients use a board subdivided in 81 square fields and figures distinguishable by gender with dots as eyes and additional cubes for different heights of the figures to describe their mental view of their family relationship. We suggest to use our iTAOs as physical representation of family members. Adding 'shoulders' and a 'nose' to the squared objects even allows to use relative orientation of the objects to other objects. Additional TUImod layers allow changing the height of the iTAOs. Therapist and patient can jointly setup a configuration which fits and the mTAO could be used to save a configuration for later review, or to restore another configuration constructed from another perspective, e.g. the family system as experienced in childhood. The therapist can then use the mTAO as described above to save the arrangement and load it for later therapy sessions or analysis.

\footnotetext{
${ }^{3}$ http://tangibleauditoryinterfaces.de/index.php/tai-applications/audiodome-soundblox/

${ }^{4}$ http://www.techfak.uni-bielefeld.de/ags/ami/publications/RHR2011-SRM/
} 


\section{Discussion and Conclusion}

In this paper we have presented a system that allows enhancing table-top TUIs with saving and restoring capabilities through a fully tangible menu metaphor. This approach allows multiple users working with the TUI to access menus equally. Our approach allows to have multiple mTAOs on the table to enable the comparison of multiple arrangements.

By using motorized potentiometers the state of the menu dial itself can be saved and restored as an inner degree of freedom for our actuated tangible objects. This is useful in future developments.

Our system is in an early state so that there is still room for technical improvements. Here for we currently create a new tracking system with custom markers that use infrared light. Furthermore we consider recording and segmenting interaction sequences and add further interaction possibilities known from GUIs such as undo and redo functions.

We also plan to implement the second application proposed in this paper. Furthermore we want to conduct a user study that proves the usability of these applications.

\section{$7 \quad$ Acknowledgements}

We thank the German Research Foundation (DFG) and the Center of Excellence 277 Cognitive Interaction Technology (CITEC) who funded this work within the German Excellence Initiative.

\section{References}

1. Audiopad: A tag-based interface for musical performance. Patten, J., Recht, B. and Ishii, H. Singapore : National University of Singapore, 2002. Proceedings of the 2002 conference on New interfaces for musical expression. pp. 1-6.

2. The reactable*. Jorda, S., et al., et al. Barcelona, Spain : s.n., 2005. Proceedings of the International Computer Music Conference (ICMC 2005), Barcelona, Spain. pp. 579-582.

3. Low-cost multi-touch sensing through frustrated total internal reflection. Han, J.Y. s.l. : ACM, 2005. Proceedings of the 18th annual ACM symposium on User interface software and technology. p. 118.

4. Tangible interfaces for remote collaboration and communication. Brave, S., Ishii, H. and Dahley, A. s.l. : ACM, 1998. Proceedings of the 1998 ACM conference on Computer supported cooperative work. pp. 169-178.

5. The actuated workbench: computer-controlled actuation in tabletop tangible interfaces. Pangaro, G. and Maynes-Aminzade, D. and Ishii, H. New York, USA : ACM, 2002. Proceedings of the 15th annual ACM symposium on User interface software and technology. pp. 181-190. 
6. Madgets: actuating widgets on interactive tabletops. Weiss, Malte, et al., et al. New York, USA : ACM, 2010. Proceedings of the 23nd annual ACM symposium on User interface software and technology. pp. 293-302.

7. Physical Objects as Bidirectional User Interface Elements. Rosenfeld, D., et al., et al. s.l. : IEEE Computer Society, 2004, IEEE Computer Graphics and Applications, pp. 44-49.

8. Riedenklau, E. TAOs - Tangible Active Objects for Table-top Interaction. [Master's thesis]. Bielefeld, Germany : Faculty of Technology, Bielefeld University, 2009.

9. Gesture Desk - An Integrated Multi-modal Gestural Workplace for Sonification. Hermann, T., Henning, T. and Ritter, H. [ed.] Antonio and Volpe, Gualtiero Camurri. Berlin, Heidelberg, Germany: Springer, 2004. Gesture-Based Communication in Human-Computer Interaction, 5th International Gesture Workshop, GW 2003 Genova, Italy, April 15-17, 2003, Selected Revised Papers. pp. 369-379.

10. TUImod: Modular Objects for Tangible User Interfaces. Bovermann, T., et al., et al. 2008. Proceedings of the 2008 Conference on Pervasive Computing.

11. Fritsch, J. and Wrede, S. An Integration Framework for Developing Interactive Robots. [ed.] D. Brugali. Berlin : Springer, 2003, Vol. 30, pp. 291-305.

12. mediaBlocks: physical containers, transports, and controls for online media. Ullmer, B., Ishii, H. and Glas, D. New York, USA : ACM, 1998. Proceedings of the 25th annual conference on Computer graphics and interactive techniques. pp. 379386.

13. TUIO: A protocol for table-top tangible user interfaces. Kaltenbrunner, M., et al., et al. 2005. Proc. of the The 6th International Workshop on Gesture in HumanComputer Interaction and Simulation.

14. Family-system-test (FAST): A three dimensional approach to investigate family relationships. Gehring, T.M. and Wyler, I.L. 4, s.l.: Springer, 1986, Child psychiatry and human development, Vol. 16.

15. Audiopad: A tag-based interface for musical performance. Patten, J., Recht, B. and Ishii, H. Singapore : s.n., 2002. Proceedings of the 2002 conference on New interfaces for musical expression. pp. 1-6. 